\title{
Atomic Force Microscopy Characterization of Ultrasound-Sensitive Nanocomposite Microcapsules
}

\author{
T. A. Kolesnikova ${ }^{a}$, B. N. Khlebtsov ${ }^{b}$, D. G. Shchukin ${ }^{c}$, and D. A. Gorin ${ }^{a *}$ \\ ${ }^{a}$ Saratov State University, Saratov, 410601 Russia \\ ${ }^{b}$ Institute of Biochemistry and Physiology of Plants and Microorganisms, \\ Russian Academy of Sciences, Saratov, 410049 Russia \\ ${ }^{c}$ Max Planck Institute of Colloids and Interfaces, D-14422 Potsdam, Germany \\ *e-mail: GorinDA@info.sgu.ru
}

\begin{abstract}
Polyelectrolyte and nanocomposite microcapsules with shells containing iron oxide $\left(\mathrm{Fe}_{3} \mathrm{O}_{4}\right.$ magnetite) nanoparticles have been obtained using the layer-by-layer polyion assembly technique. The volume fraction of nanoparticles was varied by changing the number of their layers in the shell. The dependence of the microcapsule shell thickness on its structure, that is, on the total number of polyelectrolyte and magnetite nanoparticle layers, has been studied using atomic force microscopy. An increase in the number of polyelectrolyte layers in the shell structure leads to nonlinear growth of the shell thickness. Remote control over the permeability of microcapsules was achieved by their destruction under the action of an external acoustic (ultrasound) field. It has been established that the sensitivity of microcapsules to ultrasound depends on the volume fraction of magnetite nanoparticles in the shell. The ultrasonic treatment only produces breakage of the shells, without reducing their thickness and/or changing the composition. The results of this investigation can be used for to develop systems (in particular, magnetically sensitive) for targeted drug delivery and remote controlled release in the immediate vicinity of damaged cells and tissues in an organism.
\end{abstract}

DOI: $10.1134 / \mathrm{S} 1995078008090048$

In recent years, considerable effort has been devoted to the development and investigation of systems for controlled delivery of drugs. Targeted delivery of drugs significantly increases their efficacy and allows the total drug concentration in the organism to be considerably decreased. In order to solve this task, special containers/carriers have been designed possessing preset and controlled physicochemical and mechanical properties. The function of such containers can be performed, for example, by polymeric micelles [1-4], liposomes [5], emulsions [4, 6, 7], and colloidal particles. This class also includes polyelectrolyte (PE) microcapsules obtained by the method of polyion assembly, which consists in sequential adsorption of oppositely charged PE molecules on the surface of colloidal particles (templates), followed by the dissolution and removal of the initial core [8,9]. Using this approach, it is possible to obtain capsules with a broad range of preset dimensions (from $50 \mathrm{~nm}$ to $50 \mu \mathrm{m}$ ) and a broad spectrum of shell materials. Indeed, the shells of microcapsules can be made of almost any synthetic and natural PEs [8, 10-13], lipid bilayers, inorganic nanoparticles (e.g., of silver, gold, or iron(III) oxide) [14-16], and polyvalent metal ions. The core (template) material can be selected from colloidal particles of various kinds with diameters ranging from tens of nanometers to several tens of microns [8,10,17-19]. The capsule walls can also be controlled with respect to thick- ness, functional role, and permeability to any low-and high-molecular-mass compounds [20-22].

The unique properties of PE microcapsules make it possible to use them in various fields of science and technology, such as biotechnology, cosmetics, the food industry, and medicine. The main advantage to such capsules in medical diagnostics is possibility of filling them with various drugs [23] and controlling their properties (e.g., elasticity and deformability) by varying the shell composition, template material, solvent [24], and the nature and number of PE layers. However, in order to ensure the desired result, a container must release the encapsulated drug in the immediate vicinity of damaged cells. For this purpose, inorganic magnetic nanoparticles of $\mathrm{Fe}_{3} \mathrm{O}_{4}$ (magnetite) possessing pronounced magnetic properties can be introduced in the shell composition at the synthesis stage, which makes it possible to control the motion of capsules under an applied external magnetic field. Magnetite nanoparticles also possess certain advantages from a pharmaceutical standpoint: (i) the surface of these particles can be readily modified with compounds ensuring the biocompatibility and selective adsorption of microcapsules on damaged cells; (ii) the magnetic parameters of nanoparticles make their local concentration possible with the aid of magnetic fields [25-27]. The magnetic-fieldcontrolled delivery and localization of magnetic nanoparticles with a preset magnetic transition temperature 
in the zone of a tumor underlies the method of hyperthermy [25, 27], according to which the magnetic-fielddriven particles accumulate in the tumor and are then heated to a preset temperature by an alternating magnetic field.

The permeability of microcapsule shells can be modified by various methods to facilitate the release of encapsulated drugs, for example, by varying the temperature [28] or acidity of a medium [20,21], by microwave [29] and laser radiation [14], and by application of an alternating magnetic field [15]. The effect of laser radiation is based on the heating of metal particles incorporated in the shell of microcapsules, which leads to a change in the permeability or even complete destruction of the shell and the resulting release of encapsulated drugs. However, we believe that the most promising method is based on the use of ultrasound, which is characterized by concentrated, distant, and local action. Earlier, the dependence of integrity and permeability of the shell of PE capsules on the duration and power of ultrasonic treatment and on the presence of metal nanoparticles in the shell composition was studied in [30-32]. It was established that the degree of capsule damage by ultrasound increases with the duration of treatment, this being accompanied by a decrease in the size of shell fragments [30, 31]. In addition, it was demonstrated that the ultrasonic destruction of capsules can be used for the controlled catalysis of various chemical reactions [31].

In the present study, we devoted special attention to the dependence of the sensitivity of PE microcapsules to the ultrasonic action on the content of nanoparticles in the shell, that is, on their volume fraction, which was varied by changing the number of magnetite nanoparticle layers. Prior to and after ultrasonic treatment, the microcapsules were studied by atomic force microscopy (AFM) [33]. This method, together with transmission and scanning electron microscopy, is frequently used for determining the shape of microcapsules, evaluating the shell thickness, and characterizing the surface microrelief.

\section{EXPERIMENTAL}

\section{Reagents, Instrumentation, and Methods}

The experimental samples of microcapsules were prepared using cationic and anionic PEs represented by solutions of poly(allylamino hydrochloride) (PAH) and poly(sodium styrene sulfonate) (PSS). PSS and PAH (molecular weight, $70000 \mathrm{kDa}$; concentration, $2 \mathrm{mg} / \mathrm{ml}$; Sigma-Aldrich Chemie $\mathrm{GmbH}$ ) was dissolved in $0.5 \mathrm{M}$ aqueous $\mathrm{NaCl}$ solution. Magnetite $\left(\mathrm{Fe}_{3} \mathrm{O}_{4}\right)$ nanoparticles were synthesized as described in [34] and stabilized by citric acid. The particles were negatively charged and had a predominantly spherical shape with an average diameter of $12 \mathrm{~nm}$. They were used as an aqueous suspension with a concentration of $8 \mathrm{mg} / \mathrm{ml}$. The PE and nanocomposite microcapsules were syn- thesized on polystyrene latex templates with diameters of $5.3 \pm 0.1 \mu \mathrm{m}$ (Diafarm, St. Petersburg) and 9.6 $\pm 0.1 \mu \mathrm{m}$ (Micro Particles GmbH, Germany). The process was carried out using a centrifuge mixer (Elmi CM 70M.07, EU) equipped with a 12-cell rotor for 1.5- and 2.0-ml Eppendorff tubes. All samples were prepared using twice-distilled water in two-stage purification in a BS-192 system (Russia).

The ultrasonic treatments were performed on URSK N070 setups (Russia) operating at a frequency of $27 \mathrm{kHz}$ and a power density of $3.5 \mathrm{~W} / \mathrm{cm}^{2}$.

At the synthesis stage and during subsequent treatments, the microcapsules were examined with a Biolam-70.M1 optical microscope (LOMO, Russia) equipped with a modified sample illumination system (the microscope provided a maximum magnification of up to $1800 \times$ ). The microcapsules were studied in aqueous suspensions, by placing a drop of suspension between the cover glass and the object carrier.

The surface morphology of microcapsules was studied by AFM at room temperature $\left(20-25^{\circ} \mathrm{C}\right)$ using an NT-MDT Solver-Bio instrument (Zelenograd, Russia) operating in the tapping mode. The instrument was equipped with NSG 10/20 cantilevers and provided scanning of a $60 \times 60 \mu \mathrm{m}$ area (maximum) at a frequency of $1 \mathrm{~Hz}$. The samples for AFM investigation were prepared by drying a droplet of suspension on a clean mica substrate (G250-3, 11 × $11 \mathrm{~mm}$; Plano, Germany), which was fastened on thin cover glass surface with the aid of double-sided tape (Scotch).

\section{Technology of Polyelectrolyte Microcapsules}

At the first stage, $400 \mu \mathrm{l}$ of the template suspension was placed into a tube and diluted with twice-distilled water to $2 \mathrm{ml}$. Prior to the position of the first PE layer, the templates were triply rinsed in twice-distilled water. For deposition of the first PE layer, polystyrene templates (possessing a negative initial charge) were introduced into a solution of positively charged $\mathrm{PAH}$ $(2 \mathrm{mg} / \mathrm{ml})$ in $0.5 \mathrm{M}$ aqueous $\mathrm{NaCl}$ solution. The adsorption was carried out by continuously shaking the tubes with mixtures in a minishaker for $15 \mathrm{~min}$ at $30 \mathrm{~Hz}$. This treatment ensured uniform deposition of PE molecules on the surface of colloidal particles and prevented the aggregation of these particles and their precipitation on the tube bottom. After deposition of the first PAH layer, the template suspension was centrifuged for $3 \mathrm{~min}$ at $5000 \mathrm{rpm}$ in order to separate the suspension into fractions and remove supernatant liquid phase. Then, the suspension was again triply rinsed in twice-distilled water for $3 \mathrm{~min}$ with decantation of the supernatant solution.

At the next stage, the second PE layer was formed by the absorption of negatively charged PSS molecules according to the scheme described above. Thus, after a certain number of adsorption cycles with oppositely charged PE molecules, a shell with a preset number of 
PE layers was obtained on the surface of colloidal particles.

In some part of samples, magnetite $\left(\mathrm{Fe}_{3} \mathrm{O}_{4}\right)$ nanoparticles were embedded into the shells of microcapsules. Previously, it was established [35] that, to reliably introduce magnetite nanoparticles into the shell, it is necessary to preliminarily deposit at least two PAH/PSS bilayers and to terminate the shell assembly by at least one such bilayer in order to prevent nanoparticles from being washed out at the stage of template dissolution. Since our nanoparticles were stabilized with citric acid and possessed an initial negative charge, they were added to the suspension beginning with the sixths layer instead of the PSS polyelectrolyte layer. The adsorption of $\mathrm{Fe}_{3} \mathrm{O}_{4}$ nanoparticles was carried out using a method analogous to the deposition of PE molecules. The formation of such nanocomposite microcapsules was terminated by the deposition of one or several PAH/PSS bilayers, depending on the total number of layers and the number of magnetite layers in the shell.

After the formation of a shell comprising the desired number of PE layers, the polystyrene templates were dissolved in dimethylformamide (DMF) for $48 \mathrm{~h}$. Then, the suspension of microcapsules was triply rinsed in DMF from residual polystyrene latex and, finally, it was triply rinsed in twice-distilled water and centrifuged at $7000 \mathrm{rpm}$ for $5 \mathrm{~min}$.

Using the method described above, we prepared PE microcapsules on polystyrene latex templates with diameters of $9.6 \pm 0.1$ and $5.3 \pm 0.1 \mu \mathrm{m}$, the shells of which contained various total numbers of PE layers (6, $8,10,12,14$, and 16) and various numbers of layers consisting of magnetite nanoparticles. All types of capsules were examined by means of optical microscopy and characterized by AFM prior to and after the ultrasonic treatment.

\section{RESULTS AND DISCUSSION}

The results of our experiments showed that the minimum number of PE layers necessary for the formation of stable microcapsules is ten. This result agrees well with the data reported by Dong et al. [36] for capsules synthesized on melamine formaldehyde templates. The formation of PE and nanocomposite microcapsules was monitored using an optical microscope. In the case of microcapsules with shells consisting of six and eight PE layers, the percentage of damaged capsules was rather large. This result was reproduced in repeated experimental runs. The most probable reason for an increased yield of damaged capsules is an increase in the osmotic pressure inside the capsules at the stage of template dissolution, which leads to an increase in the size of templates and a resulting fracture of relatively thin PE shells consisting of six and eight PE layers. Thus, six and eight PE layers are insufficient to ensure the stability of shells with respect to variations in the osmotic pressure in the course of template dissolution and/or with respect to mechanical loads that develop during centrifugal sedimentation of the capsules.

In contrast, it was established that the maximum number of PE layers deposited on polystyrene latex templates with diameters of $9.6 \pm 0.1$ and $5.3 \pm 0.1 \mu \mathrm{m}$ is 16 and 14, respectively. The further increase in the number of layers also led to deformation and fracture of the shells in the stage of template dissolution, which was related to an additional increase in the osmotic pressure due to a decrease in the permeability of thicker shells. This result also agrees well with the conclusions drawn in [36].

Accordingly, the maximum number of the layers of magnetic nanoparticles incorporated into the shells on microcapsules is two for ten-layer and five for 16-layer capsules, as determined by the need to deposit at least the minimum number of PAH/PSS bilayers at the initial and final stages of synthesis. Thus, we have revealed the dependence of the stability of microcapsules on the number of layers, that is, on the shell thickness.

The dependence of the thickness of the microcapsule shell on its structure (i.e., on the total number of bilayers and the number of layers consisting of iron oxide nanoparticles) was studied by AFM. The AFM investigation was performed for three groups of microcapsules synthesized on polystyrene latex templates with a diameter of $9.6 \pm 0.1 \mu \mathrm{m}$. The first group contained samples differing from each other only by the presence or absence of magnetic particles in the shell structure with the same total number of layers. Samples in the second group had different total numbers of layers $(10,12,14$, and 16) for the same number of layers of magnetic nanoparticles (two layers). In the third group, the number of layers of magnetic nanoparticles varied (from one to five) for the same total number of layers (16 layers).

Figure 1 shows the AFM images of microcapsules with the (PAH/PSS $)_{7}$ and $(\mathrm{PAH} / \mathrm{PSS})_{2}\left(\mathrm{PAH} / \mathrm{Fe}_{3} \mathrm{O}_{4}\right)_{2}(\mathrm{PAH} / \mathrm{PSS})_{3}$ structures, the latter differing from the former by the presence of magnetic particles in two layers. An analysis of these images shows that the surface morphology of capsules exhibit hardly any distinctions. This is probably related to the fact that the formation of shells with magnetite particles terminates with the deposition of at least one PAH/PSS polymer bilayer, which results in leveling (planarization) of the surface relief. For this reason, AFM does not reveal any significant difference in the surface morphology of samplers with and without nanoparticles. However, three-dimensional (3D) AFM images (Fig. 2) clearly reveal spherical superficial objects, which can represent either globular structures resulting from the formation of $\mathrm{PE}$ complexes in the presence of electrolyte in solution [37] (for microcapsules free of nanoparticles) or agglomerated nanoparticles (for the capsules containing such particles). The possibility of using PE layers for the leveling (planarization) of surfaces was demonstrated by Lvov et al. 

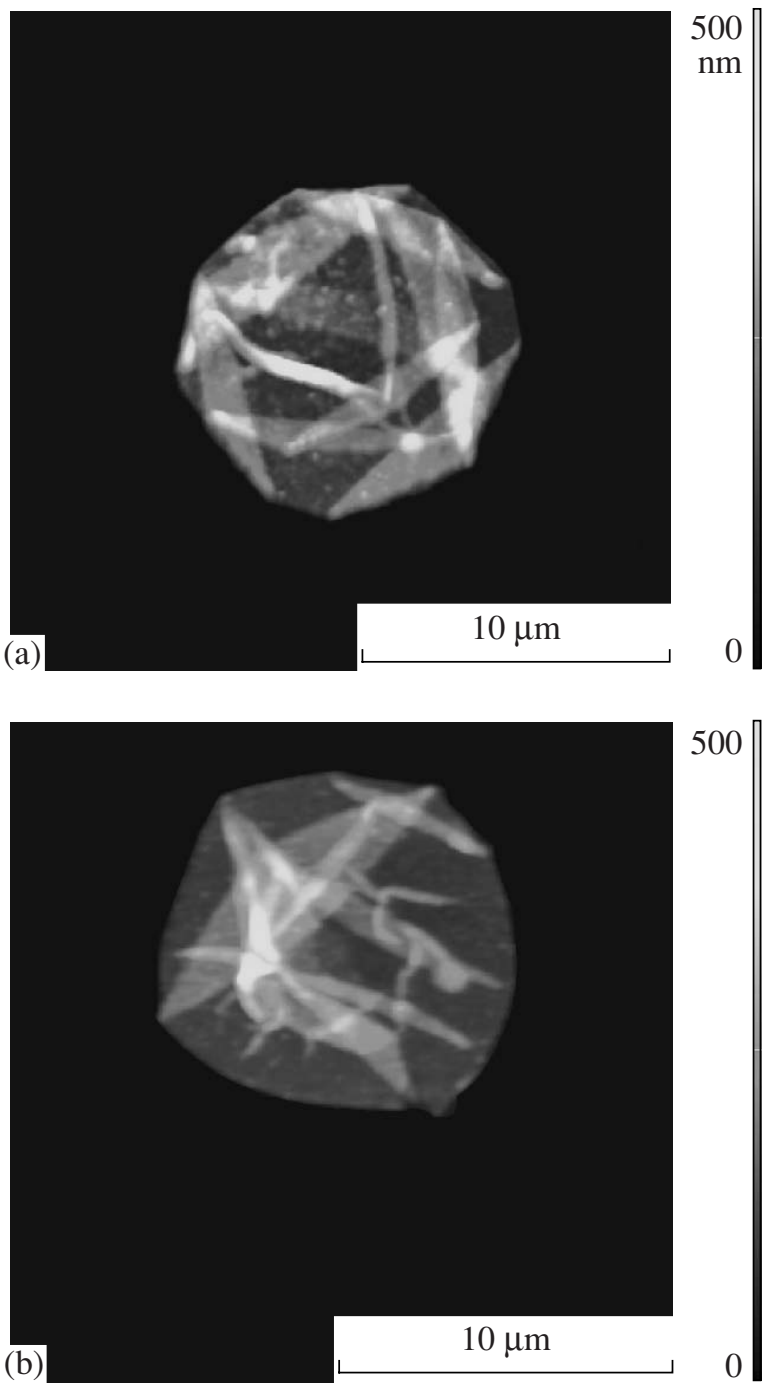

Fig. 1. AFM images of microcapsules with the (a) $(\mathrm{PAH} / \mathrm{PSS})_{7}$ and (b) $(\mathrm{PAH} / \mathrm{PSS})_{2}\left(\mathrm{PAH} / \mathrm{Fe}_{3} \mathrm{O}_{4}\right)_{2}(\mathrm{PAH} / \mathrm{PSS})_{3}$ structures.

[38] using the adsorption of viruses on a PE film, which significantly increased the roughness of its surface. The subsequent deposition of three PE bilayers led to planarization of the surface, which allowed determination of the film thickness by means of small-angle X-ray scattering.

Using the surface relief profiles reconstructed with the software of the atomic-force microscope, we determined the double shell thickness of individual capsules as the difference between the substrate (mica) surface and the thinnest part of the shell (Fig. 3). The obtained values were averaged for ten capsules from each synthesized sample.

The results of calculations showed that the introduction of magnetite nanoparticles into the shell of microcapsules leads to a decrease in its thickness compared to that in nanoparticle-free capsules with the same total
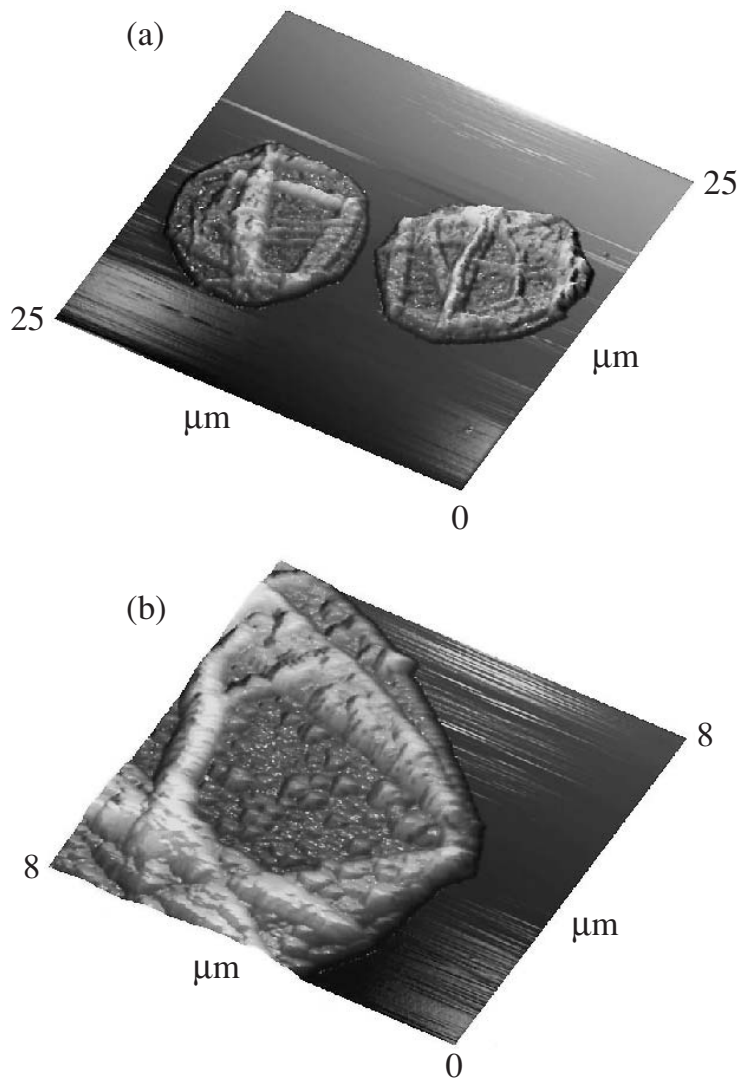

Fig. 2. 3D microcapsules with the $(\mathrm{PAH} / \mathrm{PSS})_{2}\left(\mathrm{PAH} / \mathrm{Fe}_{3} \mathrm{O}_{4}\right)_{2}(\mathrm{PAH} / \mathrm{PSS})_{4}$ structure imaged by $\mathrm{AFM}$ at (a) smaller and (b) greater magnification.

number of layers. For example, the shell thickness in 14-layer $(\mathrm{PAH} / \mathrm{PSS})_{7}$ capsules free of nanoparticles amounted to $47.2 \pm 4.4 \mathrm{~nm}$, whereas the thickness of $(\mathrm{PAH} / \mathrm{PSS})_{2}\left(\mathrm{PAH} / \mathrm{Fe}_{3} \mathrm{O}_{4}\right)_{2}(\mathrm{PAH} / \mathrm{PSS})_{3}$ capsules with two layers of magnetic nanoparticles (for the same total number of layers) was $39.1 \pm 2.9 \mathrm{~nm}$. We believe that this difference is most probably caused by a small surface charge density of magnetite nanoparticles as compared to that of PE molecules, which leads to a weaker interaction between polycation molecules and nanoparticles (compared to that between the oppositely charged PE molecules). In addition, the decrease in the shell thickness upon the introduction of nanoparticles is related to densification of the structure of microcapsules as a result of the interpenetration of nanoparticles and PE layers.

Investigation of the dependence of the microcapsule shell thickness on the number of PE layers for the same number of layers with magnetic nanoparticles revealed a nonlinear growth in the shell thickness with increasing number of PE layers. The nonlinearity is more pronounced in capsules with a smaller number of layers (Fig. 4, Table 1). These results agree well with the published data [39-41] on a nonlinear increase in the thickness of PE films on solid substrates. The nonlinear 

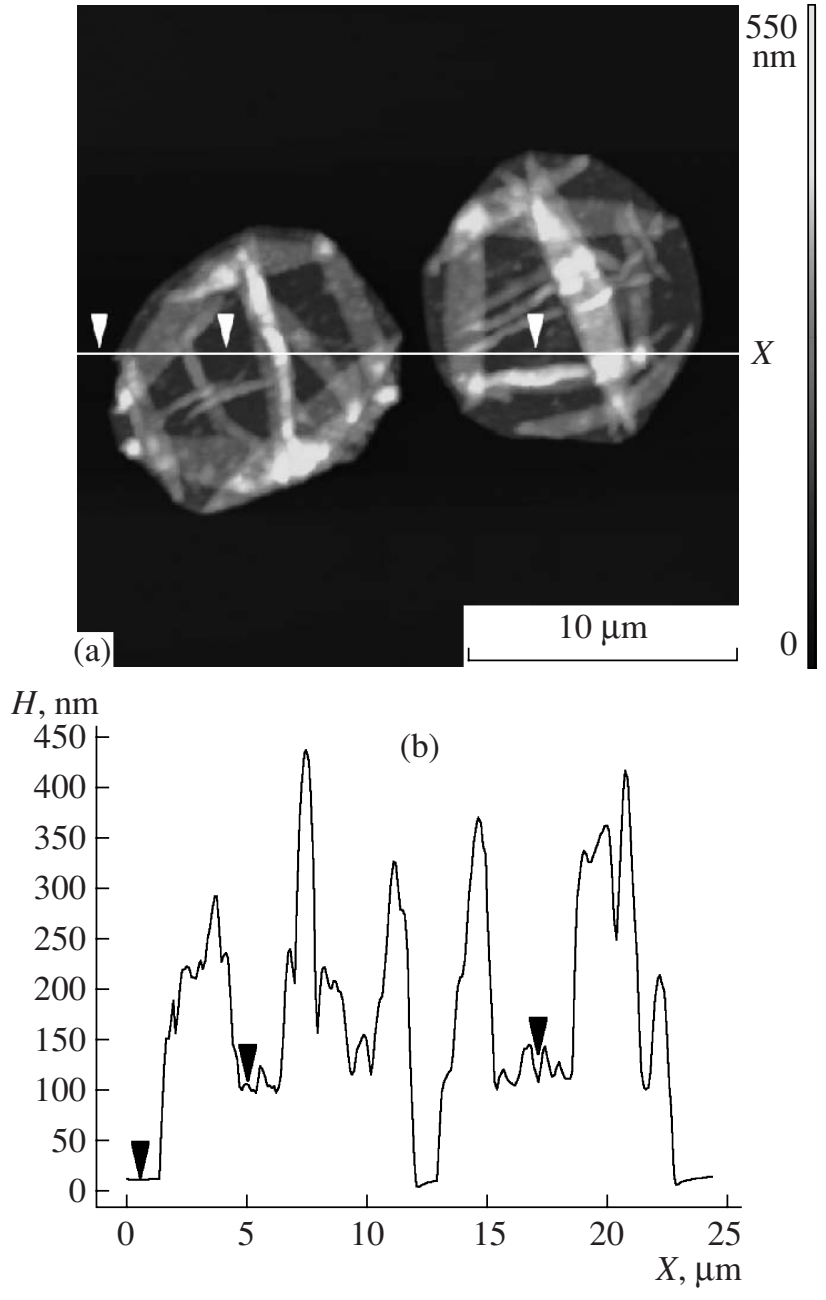

Fig. 3. Determination of the shell thickness for microcapsules with the $(\mathrm{PAH} / \mathrm{PSS})_{2}\left(\mathrm{PAH} / \mathrm{Fe}_{3} \mathrm{O}_{4}\right)_{2}(\mathrm{PAH} / \mathrm{PSS})_{4}$ structure: (a) top view; (b) surface relief profile along the line indicated in (a).

character of this dependence is related to the fact that the PE transfer in the initial stages of film formation significantly depends on the properties of colloidal particles used as templates. Since the surface charge density in colloidal particles is small, a certain part of PE molecules are desorbed in the stage of washing in twice-distilled water. As a result, the film formed in the initial stages has an inhomogeneous structure and a

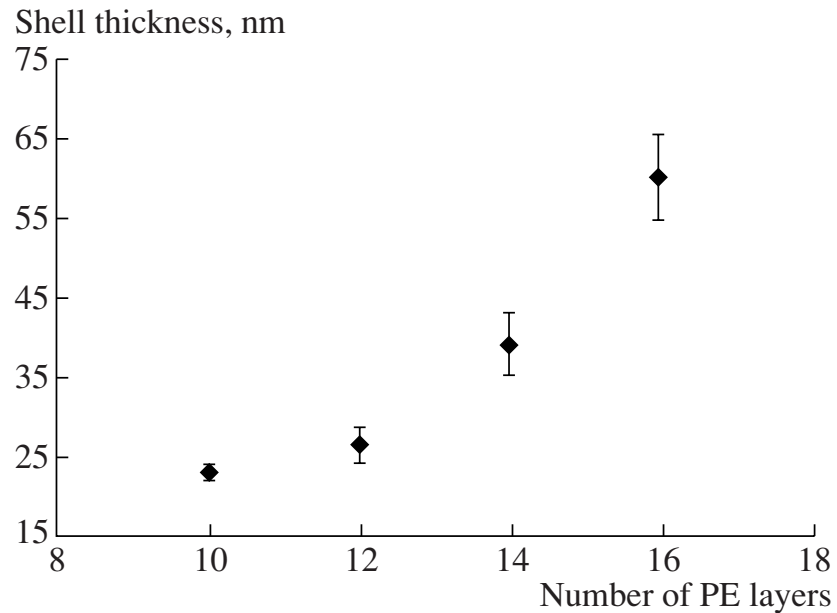

Fig. 4. Plot of the microcapsule shell thickness versus number of PE layers.

nonuniform thickness. At subsequent stages, PE molecules diffuse from the outer surface inward the film, which leads to exponential growth in the thickness. When the film thickness reaches a certain critical value, the diffusing PE molecules fail to penetrate through the entire film, equal portions of PE molecules are transferred at each step of adsorption, and the shell thickness increases linearly (rather than exponentially). The external diffusion layer has a constant thickness determined by the properties of a particular PE pair and by the conditions of film formation, independent of the number of adsorption steps. Thus, with an increasing number of layers, the PE film thickness becomes more uniform, which is accompanied by smoothing of the surface as a result of its planarization by PE layers. Analogous results were reported [42] for capsules synthesized on silicon templates using a PE pair of PSS and poly(dimethyldiallylammonium chloride)

The AFM data also showed that an increase in the number of layers of the magnetic nanoparticles is accompanied by variations in the microcapsule shell thickness. Table 2 presents data on the thicknesses of shells in 16-layer microcapsules with various numbers of magnetite nanoparticle layers. As can be seen, an increase in the number of nanoparticle layers from one to three leads to an increase in the shell thickness, but the thickness of shells containing four and five layers of

Table 1. Dependence of the microcapsule shell thickness on the number of PE layers for a fixed number (two) of layers with magnetic nanoparticles

\begin{tabular}{c|c|c}
\hline Total number of layers & Shell structure & Shell thickness, $\mathrm{nm}$ \\
\hline 10 & $(\mathrm{PAH} / \mathrm{PSS})_{2}\left(\mathrm{PAH} / \mathrm{Fe}_{3} \mathrm{O}_{4}\right)_{2}(\mathrm{PAH} / \mathrm{PSS})$ & $23.0 \pm 0.8$ \\
12 & $(\mathrm{PAH} / \mathrm{PSS})_{2}\left(\mathrm{PAH} / \mathrm{Fe}_{3} \mathrm{O}_{4}\right)_{2}(\mathrm{PAH} / \mathrm{PSS})_{2}$ & $26.5 \pm 2.3$ \\
14 & $(\mathrm{PAH} / \mathrm{PSS})_{2}\left(\mathrm{PAH} / \mathrm{Fe}_{3} \mathrm{O}_{4}\right)_{2}(\mathrm{PAH} / \mathrm{PSS})_{3}$ & $39.1 \pm 2.9$ \\
16 & $(\mathrm{PAH} / \mathrm{PSS})_{2}\left(\mathrm{PAH} / \mathrm{Fe}_{3} \mathrm{O}_{4}\right)_{2}(\mathrm{PAH} / \mathrm{PSS})_{4}$ & $60.0 \pm 5.3$ \\
\hline
\end{tabular}


Table 2. Dependence of the shell thickness in 16-layer microcapsule on the number of layers of magnetic nanoparticles

\begin{tabular}{c|c|c|c}
\hline $\begin{array}{c}\text { Total number } \\
\text { of layers }\end{array}$ & Shell structure & $\begin{array}{c}\text { Number } \\
\text { of } \mathrm{Fe}_{3} \mathrm{O}_{4} \text { layers }\end{array}$ & Shell thickness, nm \\
\hline 16 & $(\mathrm{PAH} / \mathrm{PSS})_{2}\left(\mathrm{PAH} / \mathrm{Fe}_{3} \mathrm{O}_{4}\right)(\mathrm{PAH} / \mathrm{PSS})_{5}$ & 1 & $50.8 \pm 3.5$ \\
16 & $(\mathrm{PAH} / \mathrm{PSS})_{2}\left(\mathrm{PAH} / \mathrm{Fe}_{3} \mathrm{O}_{4}\right)_{2}(\mathrm{PAH} / \mathrm{PSS})_{4}$ & 2 & $60.0 \pm 5.3$ \\
16 & $(\mathrm{PAH} / \mathrm{PSS})_{2}\left(\mathrm{PAH} / \mathrm{Fe}_{3} \mathrm{O}_{4}\right)_{3}(\mathrm{PAH} / \mathrm{PSS})_{3}$ & 3 & $65.9 \pm 1.6$ \\
16 & $(\mathrm{PAH} / \mathrm{PSS})_{2}\left(\mathrm{PAH} / \mathrm{Fe}_{3} \mathrm{O}_{4}\right)_{4}(\mathrm{PAH} / \mathrm{PSS})_{2}$ & 4 & $51.9 \pm 4.4$ \\
16 & $(\mathrm{PAH} / \mathrm{PSS})_{2}\left(\mathrm{PAH} / \mathrm{Fe}_{3} \mathrm{O}_{4}\right)_{5}(\mathrm{PAH} / \mathrm{PSS})$ & 5 & $55.2 \pm 2.3$ \\
\hline
\end{tabular}

nanoparticles is smaller than that of the shells with two and three such layers. This behavior is explained as follows. On the one hand, the shell thickness increases with the number of magnetic layers. On the other hand, this implies a decrease in the number of blocking PE layers and, hence, an increase in the probability of nanoparticle desorption from the shell in the course of template dissolution, with a resulting decrease in the shell thickness. Since these trends are competitive, the maximum shell thickness is realized for the shell structure with three magnetite layers, which is therefore optimum for 16-layer microcapsules. By blocking layers is meant those terminating the shell formation process. Figure 5 shows the plot of the thickness of a 16layer microcapsule shell versus number of layers of magnetic nanoparticles.

In order to study the effect of ultrasonic treatment on the properties of obtained PE shells, a suspension of microcapsules was exposed, for various times, to ultrasound at a power density of $3.5 \mathrm{~W} / \mathrm{cm}^{2}$. At this intensity, the action of ultrasound is mostly related to the cavitation phenomenon. Considerable energy released upon the collapse of cavitation bubbles, appearing in the immediate vicinity of microcapsules, produces breakage of their shells. The effect of ultrasound on the capsules was studied by means of optical microscopy and

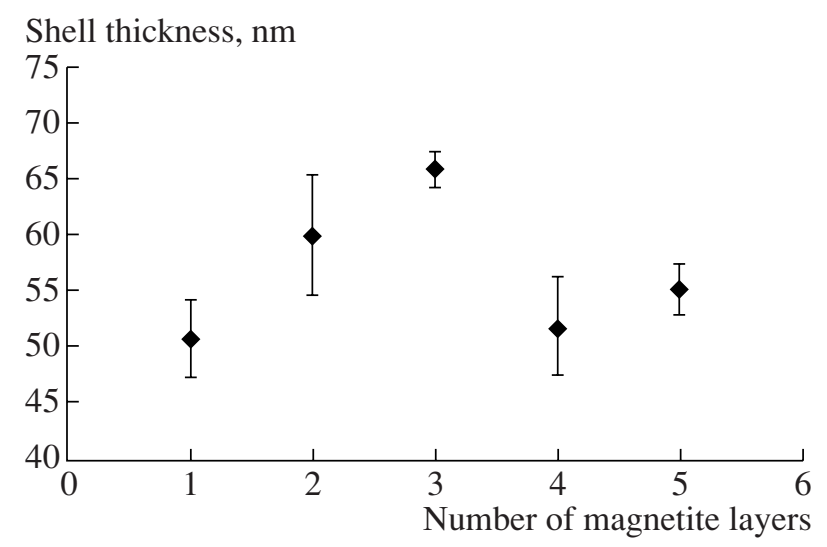

Fig. 5. Plot of the thickness of a 16-layer microcapsule shell versus total number of layers of magnetic nanoparticles.
AFM. Figure 6 demonstrates the breakage of $(\mathrm{PAH} / \mathrm{PSS})_{2}\left(\mathrm{PAH} / \mathrm{Fe}_{3} \mathrm{O}_{4}\right)_{2}(\mathrm{PAH} / \mathrm{PSS})_{2}$ nanocomposite capsules under the action of ultrasound as observed in the optical microscope at a magnification of 180x. These images show that, prior to the ultrasonic treatment, capsules had predominantly spherical shapes (with diameters corresponding to the dimensions of
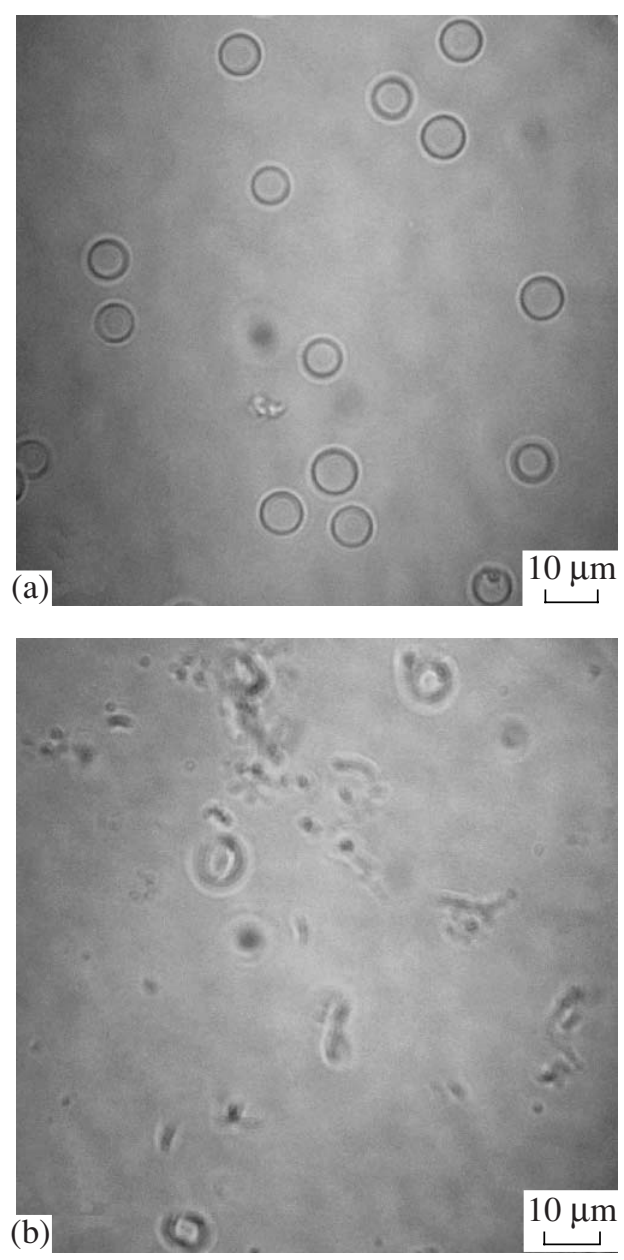

Fig. 6. Photographs of microcapsules with the $(\mathrm{PAH} / \mathrm{PSS})_{2}\left(\mathrm{PAH} / \mathrm{Fe}_{3} \mathrm{O}_{4}\right)_{2}(\mathrm{PAH} / \mathrm{PSS})_{2}$ structure (a) before and (b) after ultrasonic treatment at $3.5 \mathrm{~W} / \mathrm{cm}^{2}$. 

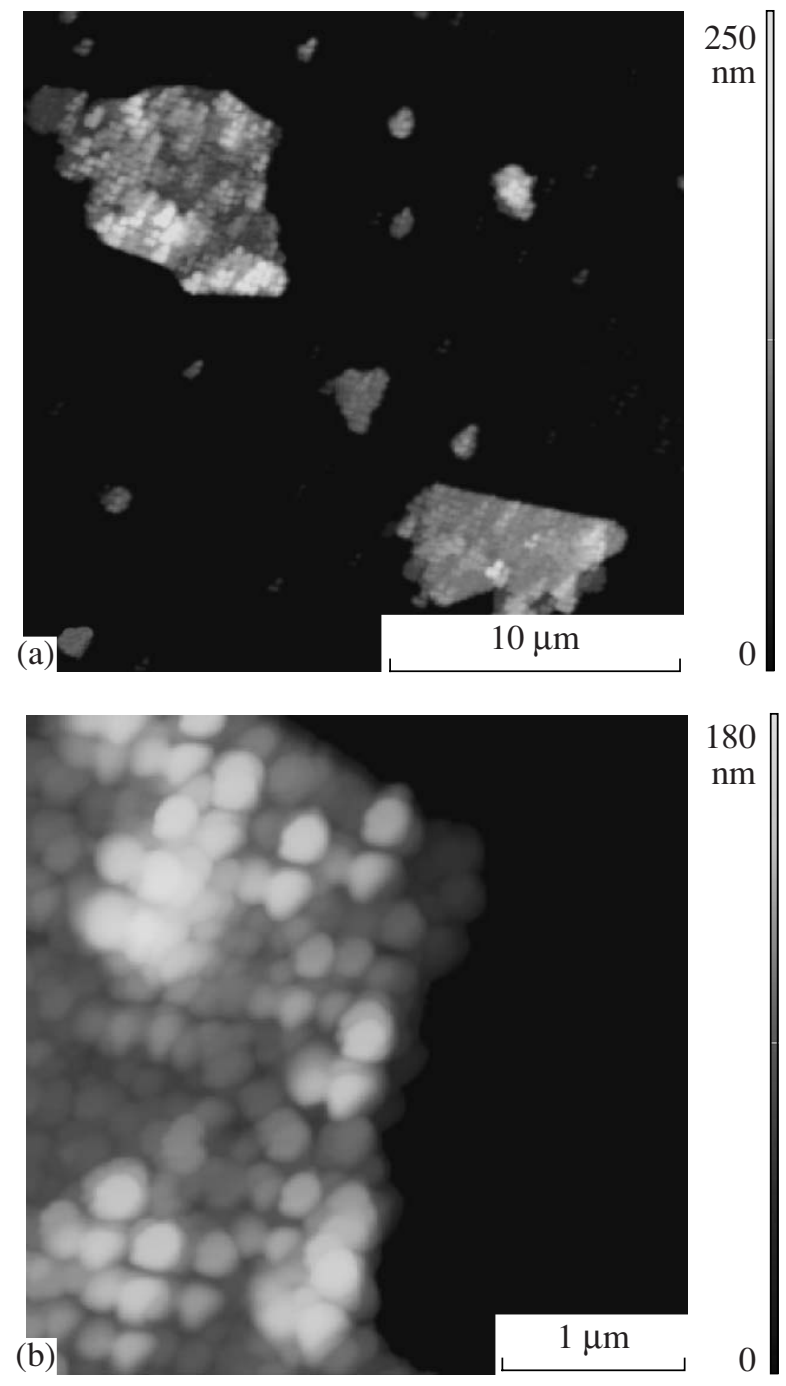

Fig. 7. Fragments of microcapsules with the $(\mathrm{PAH} / \mathrm{PSS})_{2}\left(\mathrm{PAH} / \mathrm{Fe}_{3} \mathrm{O}_{4}\right)_{2}(\mathrm{PAH} / \mathrm{PSS})_{4}$ structure after ultrasonic treatment, as imaged by AFM at (a) smaller and (b) greater magnification.

template particles), a low degree of aggregation, and homogeneous intact shells. The image obtained after treatment clearly reveals fragments of shells which formed as a result of ultrasound-induced destruction of microcapsules.

Figure 7 shows AFM images of the fragments of microcapsules with the $(\mathrm{PAH} / \mathrm{PSS})_{2}\left(\mathrm{PAH} / \mathrm{Fe}_{3} \mathrm{O}_{4}\right)_{2}(\mathrm{PAH} / \mathrm{PSS})_{4}$ structure. The shell thickness determined from the surface relief profiled by AFM was $61.3 \pm 2.7 \mathrm{~nm}$, which was consistent (to within the experimental error) with the thicknesses of shells possessing analogous structures (60.0 \pm $5.3 \mathrm{~nm}$ ). Thus, using the AFM data, we demonstrated that ultrasonic treatment only breaks the shells of microcapsules, while their surface morphology remains unchanged.

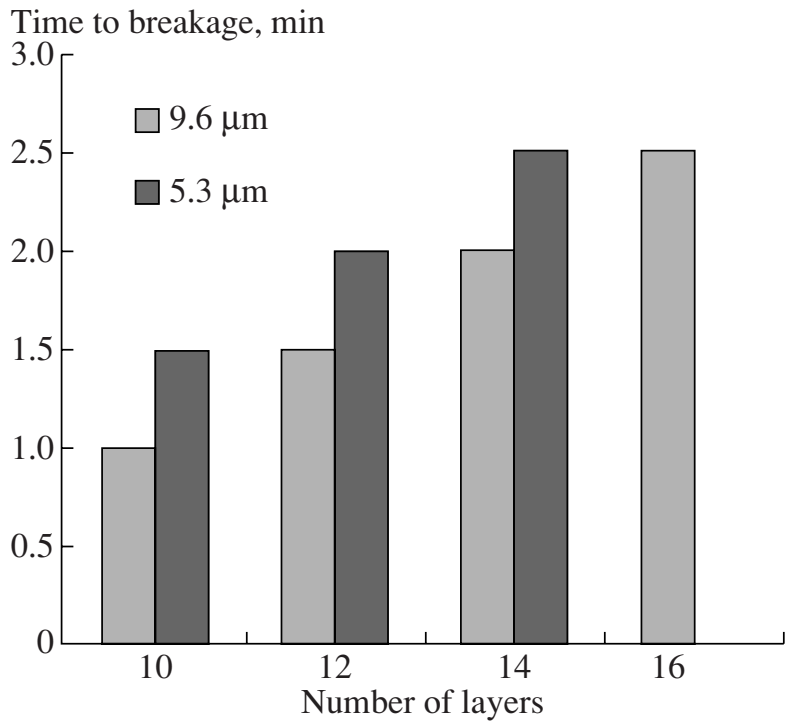

Fig. 8. Comparison of the sensitivity to ultrasound (time to complete breakage) of the microcapsules with various diameters and numbers of layers (shell thicknesses), all containing two layers of magnetic nanoparticles.

It was established that the breakage of microcapsules grown on 5.3- $\mu \mathrm{m}$ templates required a longer duration of ultrasonic treatment as compared to that necessary to break the $9.6-\mu \mathrm{m}$ capsules. The difference was about $0.5 \mathrm{~min}$ for all samples with the same number of layers of magnetic nanoparticles (Fig. 8), which is probably explained by a difference in the resistance of microcapsules with respect to the action of ultrasound, which depends on the ratio of the shell thickness to diameter. The fact that this ratio is greater for $5-\mu \mathrm{m}$ capsules than for $10-\mu \mathrm{m}$ ones accounts for the higher stability of the former. The diagram in Fig. 8 also shows that an increase in the total number of PE layers (and, hence, in their thickness) in the shell leads to an increase in the duration of ultrasonic treatment necessary for the total destruction of microcapsules.

The study of 16-layer microcapsules grown on 9.6- $\mu \mathrm{m}$ templates showed that the presence of inorganic nanoparticles in the shells significantly increased their sensitivity to ultrasound as compared to the capsules free of such nanoparticles. This can be related to the fact that, as was demonstrated above, the shell thickness in nanocomposite capsules is smaller that in nanoparticle-free PE capsules and, hence, a shorter time of ultrasonic treatment is required for the breakage of shells in the former case. In addition, the introduction of nanoparticles makes the shells inhomogeneous and, hence, less elastic and more sensitive to ultrasound. While the introduction of one layer of magnetic nanoparticles leads to a sharp increase in the sensitivity to ultrasound, the subsequent increase in the number of such layers from two to five additionally increases the time to complete breakage of the shell only from 2.5 to 4.5 min (Fig. 9), which implies that the elasticity of 


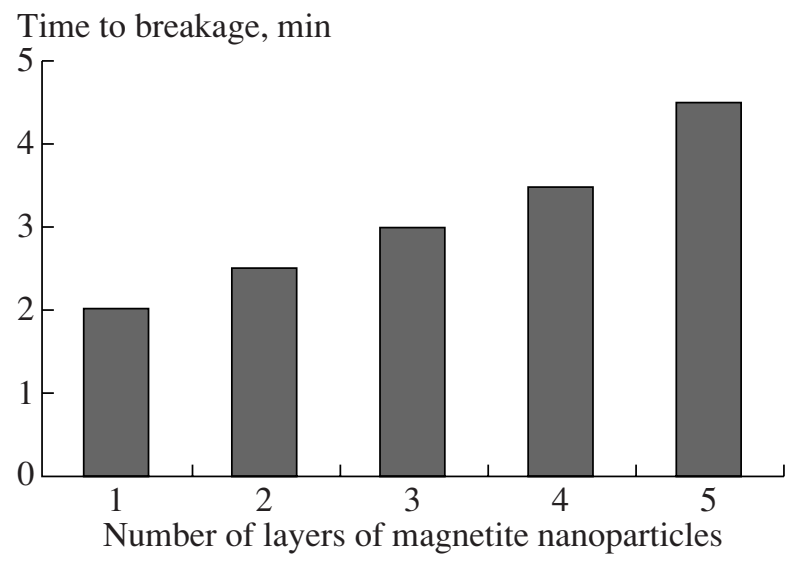

Fig. 9. Dependence of the sensitivity to ultrasound on the number of layers of $\mathrm{Fe}_{3} \mathrm{O}_{4}$ nanoparticles for 16-layer microcapsules grown on $9.6-\mu \mathrm{m}$ templates.

microcapsules increases again with an increase in the number of nanoparticles layers. This is probably related to the interpenetration of nanoparticles and PE layers in microcapsules with the increasing total volume fraction of nanoparticles in the shell, which corresponds to their more homogeneous distribution over the shell volume, rendering the medium more homogeneous and, hence, more stable with respect to the action of ultrasound.

Thus, we can ascertain that the introduction of magnetic nanoparticles in the shell composition at the assembly stage increases the sensitivity of microcapsules to ultrasound, while an increase in the total volume fraction of nanoparticles in the shell leads to their more homogeneous distribution over the shell volume, increasing its elasticity, density, and, hence, mechanical strength of microcapsules. It should be noted that these results agree well with our data reported earlier [30] concerning the effect of ultrasound on microcapsules with the $(\mathrm{PAH} / \mathrm{PSS})_{8}$ and $(\mathrm{PAH} / \mathrm{PSS})_{2}\left(\mathrm{PAH} / \mathrm{Fe}_{3} \mathrm{O}_{4}\right)_{4}(\mathrm{PAH} / \mathrm{PSS})_{2}$ structure.

\section{CONCLUSIONS}

We have described in detail the preparation of PE and nanocomposite microcapsules with shells containing iron oxide (magnetite) nanoparticles, which are sensitive to the action of ultrasound. It was demonstrated that a change in the total volume fraction of nanoparticles in the microcapsules alters their distribution in the shell and influences its elasticity, thickness, and sensitivity to ultrasound-that is, it modifies the mechanical properties of microcapsules. In this study, the volume fraction of nanoparticles was varied by changing the number of magnetite layers in the shell.

It is established that ultrasonic treatment leads to destruction of both PE and nanocomposite microcapsules, but the latter require a shorter time to complete breakage. This is related to a decrease in the elasticity of shells with inhomogeneity introduced by the nano- particle layers. However, an increase in the number of such layers leads to a more uniform distribution of nanoparticles in the shell, rendering it more homogeneous and increasing its stability under the action of ultrasound.

The AFM investigation showed that the ultrasonic treatment only produces breakage of the shells, without reducing their thickness and/or changing the composition.

Based on these results, we plan to develop a method for the remote release of encapsulated materials under the action of ultrasound. In this process, an important role is played both by the composition and structure of microcapsules and by the parameters of ultrasonic treatment, including the frequency, power, and duration. Investigation of the effect of ultrasonic treatment with various parameters on the microcapsules with PE and nanocomposite shells has both basic significance and applied interest. The proposed method can be used for to develop effective systems for targeted drug delivery and remote controlled release in the immediate vicinity of damaged cells and tissues in an organism.

\section{ACKNOWLEDGMENTS}

This study was supported in part by the joint Russian-German project (Deutsche Forschungsgemeineschaft grant RUS 113/844.0-1), the Russian Foundation for Basic Research (project no. 06-02-04009), and the Presidential Programs of Support for Young Russian Scientists (project no. MK-2637.2007.2).

\section{REFERENCES}

1. C. Allen, A. Eisenberg, and D. Maysinger, "Copolymer Drug Carriers: Conjugates, Micelles, and Microspheres," STP Pharma Sci. 9, 39-151 (1999).

2. J. X. Zhanga, L. Y. Qiua, Y. J. Zhu, and K. J. Zhu, "Physicochemical Characterization of Polymeric Micelles Constructed from Novel Amphiphilic Polyphosphazene with Poly( $N$-Isopropylacrylamide) and Ethyl 4-Aminobenzoate as Side Groups," Colloids Surf., B 43 (3-4), 123-130 (2005).

3. J. Liu, H. Lee, and C. Allen, "Formulations of Drugs in Block Copolymer Micelles: Drug Loading and Release," Curr. Pharm. Des. 12, 4685-4701 (2006).

4. D. G. Shchukin and G. B. Sukhorukov, "Nanoparticle Synthesis in Engineered Organic Nanoscale Reactors," Adv. Mater. 16 (8), 671-682 (2004).

5. D. D. Lasic, Liposomes: From Physics to Applications (Elsevier, Amsterdam, 1993).

6. X. R. Teng, D. G. Shchukin, and H. Möhwald, "A Novel Drug Carrier: Lipophilic Drug-Loaded Polyglutamate/Polyelectrolyte Nanocontainers," Langmuir 24, 383-389 (2008).

7. D. O. Grigoriev, T. Bukreeva, H. Möhwald, and D. G. Shchukin, "New Method for Fabrication of Loaded Micro- and Nanocontainers: Emulsion Encapsu- 
lation by Polyelectrolyte Layer-by-Layer Deposition on the Liquid Core," Langmuir 24, 999-1004 (2008).

8. E. Donath, G. B. Sukhorukov, F. Caruso, et al., "Novel Hollow Polymer Shells by Colloid-Templated Assembly of Polyelectrolytes," Angew. Chem., Int. Ed. 37 (16), 2201-2205 (1998).

9. D. B. Shenoy, A. A. Antipov, G. B. Sukhorukov, and H. Möhwald, "Layer-by-Layer Engineering of Biocompatible, Decomposable Core-Shell Structures,' Biomacromolecules 4, 265-272 (2003).

10. G. B. Sukhorukov, E. Donath, S. Davis, et al., "Stepwise Polyelectrolyte Assembly on Particle Surfaces: A Novel Approach to Colloid Design," Polym. Adv. Technol. 9 (10-11), 759-767 (1998).

11. G. Berth, A. Voigt, H. Dautzenberg, et al., "Polyelectrolyte Complex and Layer-by-Layer Capsules from Chitosan/Chitosan Sulfate," Biomacromolecules 3 (3), 579590 (2002).

12. N. G. Balabushevitch, O. P. Tiourina, D. V. Volodkin, et al., "Loading the Multilayer Dextran Sulfate/Protamine Microsized Capsules with Peroxidase," Biomacromolecules 4 (5), 1191-1197 (2003).

13. C. Schuler and F. Caruso, "Preparation of Enzyme Multilayers on Colloids for Biocatalysis," Macromol. Rapid Commun. 21 (11), 750-753 (2000).

14. T. V. Bukreeva, B. V. Parakhonskii, A. G. Skirtach, et al., "Preparation of Polyelectrolytic Microcapsules with Silver and Gold Nanoparticles in a Shell and the Remote Destruction of Microcapsules under Laser Irradiation," Kristallografiya 51 (5), 183-189 (2006) [Crystallogr. Rep. 51 (5), 863-869 (2006)].

15. Z. Lu, M. D. Prouty, Z. Guo, et al., "Magnetic Switch of Permeability for Polyelectrolyte Microcapsules Embedded with CoAu Nanoparticles," Langmuir 21, 20422050 (2005).

16. D. G. Shchukin, I. L. Radtchenko, and G. B. Sukhorukov, "Synthesis of Nanosized Magnetic Ferrite Particles inside Hollow Polyelectrolyte Capsules," J. Phys. Chem. B 107, 86-90 (2003).

17. G. B. Sukhorukov, E. Donath, H. Lichtenfeld, et al., "Layer-by-Layer Self-Assembly of Polyelectrolytes on Colloidal Particles," Colloids Surf., A 137 (1-3), 253266 (1998).

18. A. A. Antipov, D. Shchukin, Y. Fedutik, et al., "Carbonate Microparticles for Hollow Polyelectrolyte Capsules Fabrication," Colloids Surf., A 224, 175-184 (2003).

19. Y. Itoh, M. Matsusaki, T. Kida, and M. Akashi, "Preparation of Biodegradable Hollow Nanocapsules by Silica Template Method," Chem. Lett. 33 (12), 1552-1553 (2004).

20. A. A. Antipov, G. B. Sukhorukov, S. Leporatti, et al., "Polyelectrolyte Multilayer Capsule Permeability Control," Colloids Surf., A 198-200, 535-541 (2002).

21. G. B. Sukhorukov, A. A. Antipov, A. Voigt, et al., "pHControlled Macromolecule Encapsulation in and Release from Polyelectrolyte Multilayer Nanocapsules," Macromol. Rapid Commun. 22, 44-46 (2001).

22. B.-S. Kim and O. I. Vinogradova, "pH-Controlled Swelling of Polyelectrolyte Multilayer Microcapsules," J. Phys. Chem. B 108, 8161-8165 (2004).

23. X. Yang, X. Han, and Y. Zhu, “(PAH/PSS) 5 Microcapsules Templated on Silica Core: Encapsulation of Anticancer Drug DOX and Controlled Release Study," Colloids Surf., A 264, 49-54 (2005).

24. V. V. Lulevich, D. Andrienko, and O. I. Vinogradova, "Elasticity of Polyelectrolyte Multilayer Microcapsules," J. Chem. Phys. 120, 3822-3826 (2004).

25. A. K. Gupta and M. Gupta, "Synthesis and Surface Engineering of Iron Oxide Nanoparticles for Biomedical Applications," Biomaterials 26, 3995-4021 (2005).

26. I. Brigger, C. Dubernet, and P. Couvreur, "Nanoparticles in Cancer Therapy and Diagnosis," Adv. Drug Delivery Rev. 54, 631-651 (2002).

27. A. Jordan, R. Scholz, P. Wust, et al., "Endocytosis of Dextran and Silan-Coated Magnetite Nanoparticles and the Effect of Intracellular Hyperthermia on Human Mammary Carcinoma Cells In Vitro," J. Magn. Magn. Mater. 194, 185-196 (1999).

28. C. Gao, S. Leporatti, S. Moya, et al., "Swelling and Shrinking of Polyelectrolyte Microcapsules in Response to Changes in Temperature and Ionic Strength," Chem.-Eur. J. 9 (4), 915-920 (2003).

29. D. A. Gorin, D. G. Shchukin, A. I. Mikhailov, et al., "Effect of Microwave Radiation on Polymer Microcapsules Containing Inorganic Nanoparticles," Pis'ma Zh. Tech. Fiz. 32 (2), 45-50 (2006) [Tech. Phys. Lett. 32 (1), 70-72 (2006)].

30. D. G. Shchukin, D. A. Gorin, and H. Möhwald, "Ultrasonically Induced Opening of Polyelectrolyte Microcontainers," Langmuir 22, 7400-7404 (2006).

31. A. G. Skirtach, B. G. de Geest, A. A. Mamedov, et al., "Ultrasound Stimulated Release and Catalysis Using Polyelectrolyte Multilayer Capsules," J. Mater. Chem. 17, 1050-1054 (2007).

32. B. G. de Geest, A. G. Skirtach, A. A. Mamedov, et al., "Ultrasound-Triggered Release from Multilayered Capsules," Small 3 (5), 804-808 (2007).

33. V. L. Mironov, Fundamentals of Scanning Probe Microscopy (Tekhnosfera, Moscow, 2005) [in Russian].

34. M. Racuciu, D. E. Creanga, and Gh. Calugaru, "Synthesis and Theological Properties of an Aqueous Ferrofluid," J. Optoelectron. Adv. Mater. 7 (6), 2859-2864 (2005).

35. D. V. Andreeva, D. A. Gorin, D. G. Shchukin, and G. B. Sukhorukov, "Magnetic Microcapsules with Low Permeable Polypyrrole Skin Layer," Macromol. Rapid Commun. 27, 931-936 (2006).

36. W. Dong, J. Ferri, T. Adalsteinsson, et al., "Influence of Shell Structure on Stability, Integrity, and Mesh Size of Polyelectrolyte Capsules: Mechanism and Strategy for Improved Preparation," Chem. Mater. 17, 2603-2611 (2005). 
37. S. Katsuhiko, I. Suzuki, and J. Anzai, "Preparation of Polyelectrolyte-Layered Assemblies Containing Cyclodextrin and Their Binding Properties," Langmuir 19, 7406-7412 (2003).

38. Y. Lvov, H. Haas, G. Decher, and H. Möhwald, "Successive Deposition of Alternate Layers of Polyelectrolytes and a Charged Virus," Langmuir 10 (11), 4232-4236 (1994).

39. S. T. Dubas and J. B. Schlenoff, "Factors Controlling the Growth of Polyelectrolyte Multilayers," Macromolecules 32 (24), 8153-8160 (1999).
40. C. Porcel, P. Lavalle, V. Ball, et al., "From Exponential to Linear Growth in Polyelectrolyte Multilayers," Langmuir 22, 4376-4383 (2006).

41. R. Klitzing and H. Möhwald, "A Realistic Diffusion Model for Ultrathin Polyelectrolyte Films," Macromolecules 29, 6901-6906 (1996).

42. Karen Köhler, "Temperature-Induced Rearrangements of Polyelectrolyte Multilayer Capsules: Mechanisms and Applications," Dissertation (Max-Planck-Institut für Kolloid- und Grenzflächenforschung, Potsdam, Germany, 2006). 